\title{
A Method to Monitor Local Changes in MR Signal Intensity in Articular Cartilage: A Potential Marker for Cartilage Degeneration in Osteoarthritis
}

\author{
Josephine H. Naish ${ }^{1}$, Graham Vincent ${ }^{2}$, Mike Bowes ${ }^{2}$, Manish Kothari ${ }^{3}$, \\ David White $^{3}$, John C. Waterton ${ }^{4}$, and Chris J. Taylor ${ }^{1}$ \\ 1 Imaging Science and Biomedical Engineering, University of Manchester, Manchester, UK \\ 2 imorphics Ltd., Incubator Building, Grafton Street, Manchester, UK \\ 3 Synarc Inc., 575 Market Street, San Francisco, USA \\ 4 AstraZeneca, Alderley Park, Macclesfield, Cheshire, UK
}

\begin{abstract}
Osteoarthritis (OA) involves changes in the composition and ultimately the loss of cartilage from articulating joints. MRI has the ability to non-invasively probe the compositional integrity of cartilage, thereby potentially identifying diseased cartilage before loss occurs. In this study we have developed a technique to compare local changes in signal intensity over time in fat suppressed 3D gradient echo MR images of articular cartilage in patients with OA. We have used an Active Appearance Model (AAM) based image registration to correspond locations within the cartilage in the same individual at different times. We have applied the technique to data taken over periods of 1 and 3 years in two groups of patients with established OA of the knee. In both these studies, no significant change in total cartilage volume could be detected but we were able to observe some significant changes in signal intensity. We conclude that in a study of cartilage structure the technique can provide additional information without the overhead of extra scans.
\end{abstract}

\section{Introduction}

Osteoarthritis (OA) is one of the principle causes of disability in elderly people. While a number of interventions that may slow the progression of the disease are becoming available, research has been hampered by a lack of accurate methods for assessing changes in articular cartilage in order to monitor disease progression and response to treatment. Of the available techniques, MRI would appear to be the optimal modality as it enables the (usually) non-invasive direct visualization of hyaline cartilage.

Techniques for accurately quantifying the volume of articular cartilage using fat suppressed 3D gradient echo magnetic resonance imaging have been developed and validated [1]. However, recent studies have cast doubt on the effectiveness of total cartilage volume as a measure of cartilage degeneration in OA [2] and suggest that future work should concentrate on assessing focal changes e.g. by mapping cartilage thickness. MRI also has the unique ability to non-invasively probe the compositional integrity of cartilage, thereby potentially identifying diseased cartilage before loss occurs. Measurements of cartilage quality may thus provide methods to monitor early disease progression and treatment response. These measurements include surrogates for hydration such as signal 
intensity, $T_{2}$ [3] and proton density [4], surrogates for protoglycan such as dGEMRIC [5] (which is an invasive technique) and sodium [6] (which requires a sodium coil), and surrogates for collagen such as magnetisation transfer [7]. Of these the simplest and hence most clinically useful techniques are those that monitor cartilage hydration. Several studies have shown that $T_{2}$ relaxation, measured using a multi-echo sequence, correlates with collagen matrix organisation and water content [3,89] but longitudinal studies have yet to be published. Calculation of absolute proton density requires multiple image acquisitions resulting in very long imaging times or compromising on image resolution. The technique also suffers from increased noise since it involves the calculation of secondary data sets from several primary acquisitions.

The raw signal intensity in a single fat suppressed gradient echo image is proportional to the proton density (and so related to hydration) but also affected by both $T_{1}$ and $T_{2}^{*}$ relaxation, both of which may change with cartilage degeneration. Signal intensity does not therefore provide a direct measure of hydration but may still provide a marker for change in cartilage composition. The advantage of a signal intensity measurement is that, unlike other measurements of cartilage quality, it can be obtained from the structural scans so that with no additional scan time both the regional thickness and the cartilage composition may be assessed.

In this study we have developed an Active Appearance Model (AAM) based technique to compare local changes in signal intensity over time. We have applied the technique to data collected over 1 and 3 years in two groups of patients with established $\mathrm{OA}$ of the knee in order to investigate the usefulness of signal intensity as a marker for disease progression in OA.

\section{Methods}

\subsection{Imaging}

We have used data from two separate longitudinal studies of cartilage volume in patients with OA. In the first, eleven patients with established OA of the knee were imaged at baseline, 2 months, 1 year and 3 years using a 3D spoiled gradient-echo sequence with fat suppression. The imaging was performed using a 1.0T clinical scanner and the image sequence parameters were $\mathrm{TR}=50 \mathrm{~ms}, \mathrm{TE}=11 \mathrm{~ms}, 40^{\circ}$ flip angle, sagittal slices, slice thickness $1.56 \mathrm{~mm}$, in-plane resolution $0.55 \mathrm{~mm}$ [2]. An example MR image from this data set is presented in Fig.1. Volume measurements, made using a manual segmentation of the images at each time point, yielded no significant change over the three years in any of the cartilage compartments. See [2] for full details of the volume analysis.

The second study consisted of 50 patients, imaged at baseline, 3 months, 6 months and 1 year with some repeated measurements at the 3 and 6 month time points. Imaging was performed using a $1.5 \mathrm{~T}$ clinical scanner and the sequence parameters were were $\mathrm{TR}=58 \mathrm{~ms}, \mathrm{TE}=6 \mathrm{~ms}, 40^{\circ}$ flip angle, sagittal slices, slice thickness $2 \mathrm{~mm}$, in-plane resolution $0.63 \mathrm{~mm}$. Volume measurements were made using a semi-automatic segmentation method based on the livewire algorithm. Again, no significant changes in volume could be detected over the timescale of the study. 


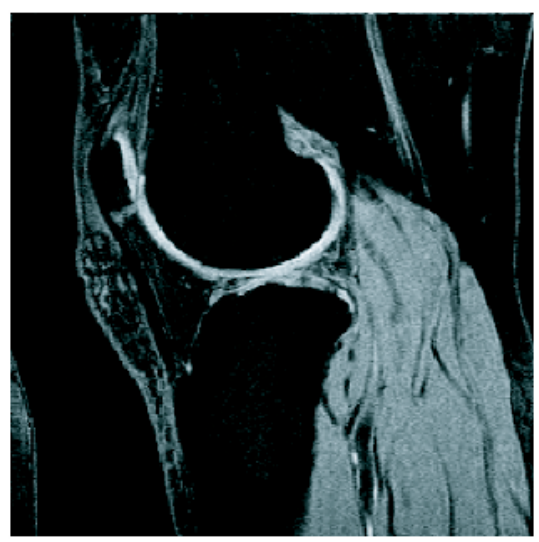

Fig. 1. Example 3D fat suppressed gradient echo magnetic resonance image. Slice shown is in the region of the lateral condyle. Note the hyper-intense signal in the regions of hyaline cartilage.

\subsection{Image Registration}

An image registration method was developed in order to compare the signal intensity at corresponding locations within the cartilage of the same individual at different times. An overall rigid registration (i.e. a global translation plus rotation) was not possible because the relative positions of the components of the knee joint can change as the knee flexes. However, a separate rigid registration for each cartilage compartment (femoral, patellar, tibial) provided a good approximation. This is only an approximation since cartilage may swell or be lost over time. Segmentations of each cartilage compartment for the data from both studies had been performed previously, either using a fully manual or semi-automated technique, as part of the cartilage volume calculations. Segmentations of the cartilage in the baseline images were used to construct a triangulated surface for each cartilage compartment. An example surface is presented in Fig. [ 2 for the femoral cartilage. For details of the surface building see [10]. Using this surface and the image signal intensities, a single example AAM [11] was built for each cartilage compartment for each individual. The models were then used to search the later time point images of each individual resulting in a translation and rotation for each compartment.

The registration was performed using Endpoint, a commercially available image analysis package written in a collaboration between the University of Manchester and imorphics.

\subsection{Analysis}

The image intensity was sampled at a set of corresponding points in each of the registered images using linear interpolation. In initial experiments these points were chosen as the grid of voxel locations in the baseline image ('rigid sampling') corresponding to the area inside the segmentation but in more recent experiments we have developed a method to sample at equidistant points along normals to the medial surface of the triangulation 


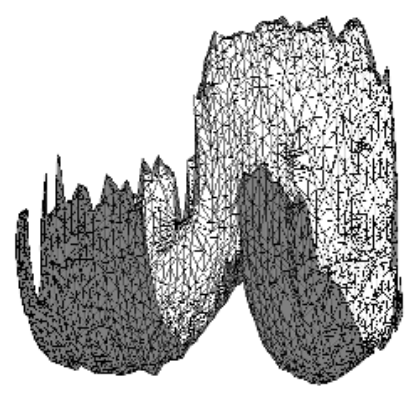

Fig. 2. Example triangulated surface built for the femoral cartilage compartment

('thickness sampling'). The final step was to correct for an overall scaling factor caused by changes in scanner settings. This was achieved by performing a robust fit (least median orthogonal distance) of a pair of sets of corresponding signal intensities. Once the scaling factor was determined in this way, differences in signal intensity over time could be investigated.

A qualitative local analysis was possible by comparing corresponding slices through the normalised 3D signal intensity at different times. We have also produced 2D maps of the signal intensity differences summed along normals to the medial surface which allow an overall spatial comparison of a cartilage segment over time.

For a more quantitative analysis, a global measure of signal intensity change was calculated by taking the average normalised difference for each cartilage compartment. The normalisation method we have used, based on a robust fit to corresponding pairs of intensity values, does not completely balance the mean signal intensity differences. The remaining global change in intensity tells us something about changes in the outliers of the distribution. These will include voxels subject to varying degrees of partial volume averaging but will also include those voxels for which a real change in signal intensity has occurred. Differences in signal intensity due to, for example, partial volume changes or differences in position in the coil will vary randomly over time whereas real differences in cartilage composition will change in some consistent way. Therefore, average net changes over time have been calculated by performing a linear regression of the average normalised signal intensity against time for each of the separate cartilage compartments for each individual. This results in values of percentage net change in total signal intensity per year.

\section{Results}

\subsection{Signal Intensity Maps}

Example results for a single slice through the cartilage are presented in Fig. 33. These images demonstrate the effectiveness of the registration process and allow local comparisons of signal intensity and so cartilage quality to be made. Some focal changes can be seen in the tibial plateau in this example. 
a)

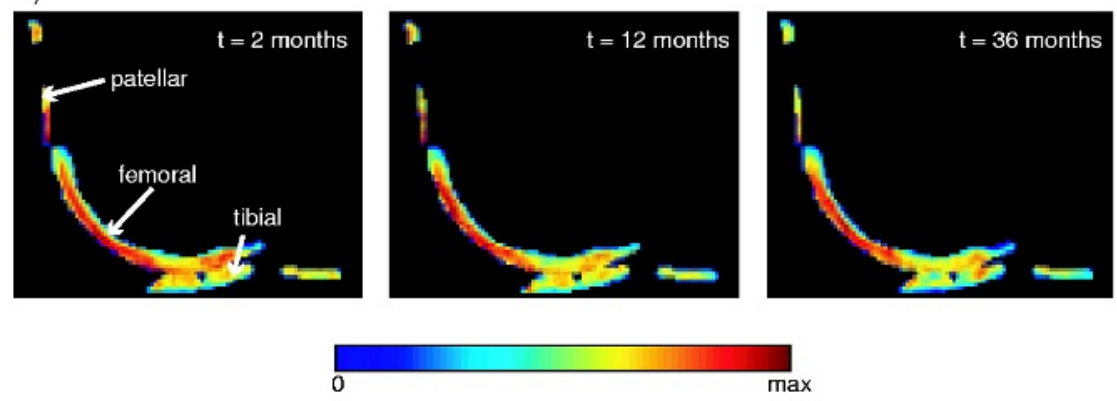

b)
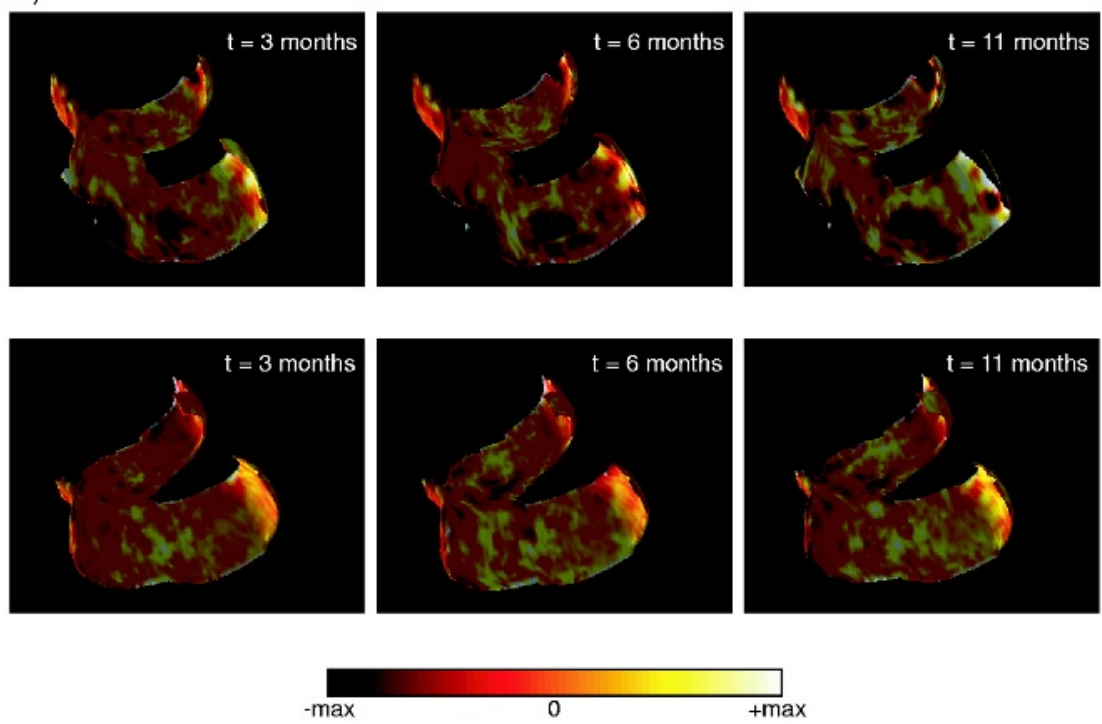

Fig. 3. a) Slice through registered and normalised cartilage compartments for three time points. b) $2 \mathrm{D}$ maps of signal intensity differences (i.e. signal intensity at time $\mathrm{t}$ minus baseline signal intensity) calculated by integrating along normals to the medial surface for the femoral cartilage compartment.

Two example 2D signal intensity difference maps are presented in Fig. 3 b for the femoral cartilage compartment. The first example shows clear evidence of disease progression in the form of increasing regions of negative signal intensity change in both femoral condyles. In the second example an overall progression is more difficult to identify but some similar structures can be seen in the maps suggesting that regions of cartilage are changing in composition in some consistent manner over time. 


\subsection{Global Measures}

For the 11 patients over 3 years two data sets could not be used because of problems with the images at one of the time points. For the 9 complete sets the following results were obtained using the rigid sampling method to sample corresponding pairs of values of signal intensity. Results are expressed as average percentage change per year (mean \pm standard error):

$\begin{array}{ll}\text { femoral } & -0.41 \pm 0.19(P=0.07) \\ \text { patellar } & -0.92 \pm 0.78(P=0.3) \\ \text { lateral tibial } & -0.22 \pm 0.10(P=0.06) \\ \text { medial tibial } & -0.65 \pm 0.34(P=0.09)\end{array}$

To obtain these results we have not used the baseline image data in the linear regression to avoid introducing any bias. The probabilities quoted are for a two-tailed Student's t-test. For all four compartments we observe a mean decrease in overall signal intensity but in no single compartment does this reach statistical significance at a 5\% level, perhaps due to the small number of individuals in this study. Note that the standard error is much larger in the case of the patellar cartilage than the other compartments; this is probably due to a combination of the increased error in registration because of the shape of this compartment and the fact that the patella tends to sit near the edge of the knee coil where the receive sensitivity is less homogeneous.

In the larger data set of 50 patients over one year we have 42 complete usable data sets. We have analysed these using the thickness sampling method to sample image intensities. The results for the femoral compartment show an annual percentage change in signal intensity of $-1.02 \pm 0.29(P<0.01)$ and in this case the result is significant statistically. This result is larger in magnitude but consistent with the results obtained for the small data set of 9 patients over three years. In order to check for systematic errors over time we have also performed a reverse experiment on this data set. Manual segmentation of the final time point (12 months) image was performed and used to construct the surfaces and build the models. The earlier time point images were then registered and normalised using this as the baseline. This has been carried out for 28 subjects. Linear regression produces an annual percentage change in signal intensity of $-1.15 \pm 0.39$ which is consistent with the results from the forward experiment and so rules out a systematic error.

\section{Discussion}

By using an AAM based image registration we are able to correspond locations within the cartilage of the same individual in MR images taken at different times. Combined with normalisation, this allows a direct monitoring of the signal intensity of a region of cartilage over time. Manual (or semi-automatic) segmentation has to be performed at a single time point only, so that user input is minimised and errors resulting from inconsistent segmentation are eliminated. Focal changes in the structure of the cartilage can be directly visualised as focal changes in signal intensity. 
By integrating the signal intensity change across normals to the medial surface of the cartilage compartments, information on the depth variation of signal intensity change is lost but the spatial distribution across the articulating surface may be more easily visualised. It is possible to identify regions of cartilage which change progressively and consistently over time suggesting that the method allows local changes in the composition of cartilage to be observed. One limitation of the technique presented is that in using a robust fit to the voxel signal intensities as the normalisation method we are assuming that the majority of the voxels within the cartilage do not change. If this assumption breaks down, i.e. if there is extensive and rapid disease progression, the method will tend to underestimate the overall change. This may be the cause of the regions of apparently increased signal intensity in the first example in Fig. $3 \mathrm{~b}$. This may be avoided in future studies by placing a signal intensity phantom in the field of view of the image and using this as a standard to calculate the scaling factor.

In order to carry out a more quantitative statistical analysis of the spatial variation of signal intensity change it will be necessary to correspond areas of cartilage across a population. A method to achieve this by modelling the underlying bone using statistical shape models has been presented in the context of cartilage thickness measurement [10] and we intend to apply this method to signal intensity measurement in a future study.

A statistical analysis of signal intensity change for this study has been carried out by calculating the global change for a cartilage compartment. We find a significant decrease in signal intensity over one year in 42 patients with established osteoarthritis. This change may be due to a reduction in hydration and hence proton density, changes in the structure (eg. collagen content) of the cartilage, an actual loss of cartilage or a combination of these factors. In future studies, compositional changes and cartilage loss may be separated by combining measurement of signal intensity with a measurement of local cartilage thickness, both of which may be obtained from a single MR scan.

\section{Conclusions}

Local signal intensity appears to offer important additional information in a study of cartilage quantity. The signal intensity in a gradient echo image is proportional to proton density (and hence cartilage hydration) but is also affected by $T_{1}$ and $T_{2}^{*}$, both of which may change with cartilage degeneration in osteoarthritis. It is therefore a non-specific marker for changes in cartilage composition but nevertheless should prove useful particularly when combined with studies of regional cartilage thickness.

Acknowledgements. We would like to thank the University of Bristol and GSK for the data used in this study. The work was supported by AstraZeneca, Synarc and imorphics.

\section{References}

1. Peterfy CG, van Dijke CF, Janzen DL, Gluer CC, Namba R, Majumdar S, Lang P, and Genant HK. Quantification of articular cartilage in the knee with pulsed saturation transfer subtraction and fat-suppressed mr imaging: optimization and validation. Radiology, 192(2):485-91, 1994. 
2. Gandy SJ, Dieppe PA, Keen MC, Maciewicz RA, Watt I, and Waterton JC. No loss of cartilage volume over three years in patients with knee osteoarthritis as assessed by magnetic resonance imaging. Osteoarthritis Cartilage, 10(12):929-37, 2002.

3. Lüsse S, Claassen H, Gehrke T, Hassenpflug J, Schunke M, Heller M, and Gluer CC. Evaluation of water content by spatially resolved transverse relaxation times of human articular cartilage. Magn Reson Imaging, 18(4):423-30, 2000.

4. Selby K, Peterfy CG, Cohen ZA, Ateshian GA, Mow VC, Roos M, Wong S, Newitt DC, van Dijke CJ, Wendland M, and Genant HK. In vivo MR quantification of articular cartliage water content: a potential early indicator of arthritis. Proc Intl Soc Magn Reson Med, 3:204, 1995.

5. Gillis A, Gray M, and Burstein D. Relaxivity and diffusion of gadolinium agents in cartilage. Magn Reson Med, 48(6):1068-71, 2002.

6. Shapiro EM, Borthakur A, Gougoutas A, and Reddy R. 23Na MRI accurately measures fixed charge density in articular cartilage. Magn Reson Med, 47(2):284-91, 2002.

7. Kim DK, Ceckler TL, Hascall VC, Calabro A, and Balaban RS. Analysis of watermacromolecule proton magnetization transfer in articular cartilage. Magn Reson Med, 29(2):211-5, 1993.

8. Smith HE, Mosher TJ, Dardzinski BJ, Collins BG, Collins CM, Yang QX, Schmithorst VJ, and Smith MB. Spatial variation in cartilage T2 of the knee. J Magn Reson Imaging, 14(1):50-5, 2001.

9. Liess C, Lusse S, Karger N, Heller M, and Gluer CC. Detection of changes in cartilage water content using MRI T2-mapping in vivo. Osteoarthritis Cartilage, 10(12):907-13, 2002.

10. Williams TG, Taylor CJ, Gao ZX, and Waterton JC. Corresponding articular cartilage thickness measurements in the knee joint by modelling the underlying bone. Lect Notes Comput Sc, 2732:126-135, 2003.

11. Cootes TF, Edwards GJ, and Taylor CJ. Active Appearance Models. IEEE Transactions on Pattern Analysis and Machine Intelligence, 23(6):681-685, 2001. 